\title{
Induction of Human Anti-Human Antibody Responses (Ab2) after Application of a Humanized Lewis $Y$ Carbohydrate Specific Antibody (Ab1): Connection of Prolonged Disease Stabilization with Ab3 Induction?
}

\author{
Andreas Nechansky ${ }^{1,2,3^{*}}$, Stefan Stranner ${ }^{1,4}$, Oliver Scheiber $^{1}$, Nicole Halanek ${ }^{1,4}$, Ralf Kircheis ${ }^{1,5}$ \\ ${ }^{1}$ Igeneon GmbH, Vienna, Austria; ${ }^{2}$ Meridian Biopharmaceuticals GmbH, Vienna, Austria; ${ }^{3}$ Vela Laboratories, Vienna, Austria; \\ ${ }^{4}$ Apeiron Biologics AG, Vienna, Austria; ${ }^{5}$ Virologik GmbH, Erlangen, Germany. \\ Email: ${ }^{*}$ andreas.nechansky@meridian-biopharm.at
}

Received June 22 $2^{\text {nd }}, 2012$; revised July 24 $4^{\text {th }}, 2012$; accepted August $3^{\text {rd }}, 2012$

\begin{abstract}
Purpose: Detailed analysis of a patient with epithelial Lewis $\mathrm{Y}(\mathrm{LeY})$ positive cancer who received twice $50 \mathrm{mg}$ of the humanized Lewis Y carbohydrate specific mAb IGN311 and developed a clinically significant human anti-human antibody (HAHA) response (Ab2). Results: Clinical stabilization of the disease was assigned to in this patient. The HAHA response consisted mainly of IgG1 and was found to be directed against the IGN311 binding site. Consistent with the induction of the HAHA response, CDC activity against Lewis Y positive target cells was completely abolished at day 8 and could not be restored by the second $50 \mathrm{mg}$ infusion indicating complete neutralization of applied IGN311. The ADCC reactivity was also significantly reduced and anti-anti idiotype-specific antibodies (Ab3) were detectable at day 65. Conclusions: Induction of Ab3 antibodies should be considered as an additional factor influencing the efficacy of humanized antibodies. In this context, the potential threat of induced HAHA responses against therapeutic mAbs might have to be reconsidered because they might actually have also beneficial immunological long-term effects leading to an active immunization component induced by therapeutic antibodies.
\end{abstract}

Keywords: Lewis Y Carbohydrate; Immunotherapy; Immunogenicity; Anti-Idiotype; HAHA (Human Anti-Human Antibodies)

\section{Introduction}

The unwanted immunogenicity of protein therapeutics is of general concern regarding patient safety and efficacy [1]. Repeated application of therapeutic murine antibodyies has been frequently found to be associated with the induction of human anti-mouse antibodies (HAMA) [2] which is unwanted because it can lead to rapid clearance of the therapeutic $\mathrm{mAb}$ or to hypersensitivity reactions. On the other hand, HAMA may be associated also with a positive clinical outcome [3,4]. Humanization of murine antibodies is regarded as versatile approach to minimize the immunogenic potential of therapeutic antibodies [5]. Nevertheless, also after application of humanized mAbs, the induction of human anti-human antibodies (HAHA) has been reported [6]. Whereas the immunological implications of the HAMA response have been investigated in great detail [7-9], the potential therapeutic effect of the HAHA response is largely unknown. In this regard the

"Corresponding author. induction of anti-idiotypic network responses may even be involved in mediating effector mechanisms [10-12]. These effects can be explained based on the anti-idiotypic network theory postulated by Jerne [13].

IGN311 is a Lewis Y (LeY) specific, humanized monoclonal $\operatorname{IgG} 1 / \kappa$ antibody that has been used for passive immunotherapy of patients suffering from cancers that are over-expressing the LeY carbohydrate antigen. To determine safety, tolerability, pharmacokinetics, and antitumor activity of IGN311, a clinical Phase I study with twelve patients with LeY positive tumors was performed [14]. After two IGN311 infusions (at days 1 and $15), 50 \%$ of the patients developed a HAHA response as determined by a surface plasmon resonance (SPR) based assay [15]. The peak response was measured at day 15 after the first infusion (before the second infusion) and then decreased steadily to almost baseline levels as measured before treatment. This kind of HAHA profile has been categorized as "Type I response" [16] and is commonly not regarded dangerous for the patient. 
We have investigated and discuss here in detail the immunological parameters and clinical outcome of one patient with an LeY expressing tumor who was treated with IGN311 (50 mg dosing group) and developed a particularly high HAHA response accompanied by a longterm stabilization of disease [14].

\section{Material and Methods}

\subsection{Patients}

All sera analyzed had been taken from patients participating in a randomized clinical Phase I trial with IGN311 [14]. Informed consent was obtained from each subject. Patient 1 (Male, 78 years, KPS: 80) and patient 2 (Male, 66 years, KPS: 70) belonged to the cohort that received twice $50 \mathrm{mg}$ IGN311 infusion per dose. Because patient 1 showed stable disease, an additional third and fourth infusion of IGN311 on days 108 and 123 at the discretion and responsibility of the investigator was given. Both patients suffered from colorectal cancer which had spread to the liver. Prior to IGN311 treatment they did not receive immunotherapy or hormonal treatment but chemotherapy. Patient 1 was treated with capecitabine and patient 2 received a combination consisting of capecitabine, folic acid and 5-flourouracil.

\subsection{Antibodies}

Humanized mAb IGN311 (IgG1/א) [17], specific for the tumor-associated carbohydrate LeY, was used both for application in patients and in vitro tests. As isotype control Ab, Herceptin was used. MMA383 (murine IgG2a), recognizing the idiotype of IGN311, was regarded as mimic of an anti-idiotypic $\mathrm{Ab} 2$ and used for the quantitation of the induced HAHA response. ABL364, the murine $\operatorname{IgG} 3 / \kappa$ variant of IGN311, was used to demonstrate that the induced HAHA response is idiotype-specific. MMA383 and ABL364 were obtained from Novartis, Vienna, Austria. As isotype control, unrelated murine $\mathrm{IgG} 3 / \kappa$ mAb FLOPC-21 (Sigma) was used.

\subsection{Determination of Human Anti-Human Antibody Response}

Analysis was performed as described applying a SPR approach using a Biacore 3000 [15]. Briefly, IGN311 was covalently immobilized onto the surface of CM5 sensor chip and 1:10 diluted serum samples from patients were tested. Unspecific binding was assessed using a flow cell where an isotype matched control antibody (Herceptin) was immobilized. A calibration curve was recorded using a murine antibody (spiked into 10\% human serum pool) that binds to the IGN311 idiotype.

\subsection{Protein G Chromatography}

Patient 1 immune sera were diluted and applied to Protein $\mathrm{G}$ columns. The flow through was collected and size exclusion chromatography (SEC) was used to confirm depletion of IgG. The area under the curve corresponding to serum albumine peak was used to adjust the concentration of the immune serum to the flow-through sample. Samples were then analysed by SPR.

\subsection{Anti-Idiotype ELISA}

Binding of pre-serum (day 1) and immune serum (day 15) of patient 1 to ABL364 - the murine $\operatorname{IgG} 3 / \kappa$ variant of IGN311-was measured. As isotype control, an unrelated murine IgG3/ $\kappa$ (FLOPC-21, Sigma) was used. Samples were serially diluted and bound Abs were detected with a polyclonal anti-human IgG-HRP.

\subsection{Idiotype Determination of the Anti-Id Response (ELISA)}

Isotype determination of human serum antibodies bound to ABL364 was performed with human IgG subclassspecific antibodies conjugated to HRP (Sigma). Total $\operatorname{IgG}$ was determined with polyclonal anti-human IgGHRP. Serum samples were diluted 1:300 and 1:2700.

\subsection{Determination Serum IGN311}

Antibody MMA383 was used to capture IGN311 present in patient serum at the indicated time points (hours) 0 (day 1, 1st infusion), 0.5, 4, 8, 24 (day 2), 48 (day 3), 96 (day 5), 168 (day 8), 336 (day 15, 2nd infusion), 336.5, 340, 344, 360 (day 16), 384 (day 17), 432 (day 19), 504 (day 22), 672 (day 29), and 1008 (day 43). Sera were diluted 1:200 and bound antibodies were detected with an anti-human IgG-HRP conjugate specific for the $\kappa$ light chain.

\subsection{Complement Dependent Cytotoxicity (CDC)}

Briefly, LeY positive SKBR3 tumor cells were incubated with $\mathrm{Na}_{2}{ }^{51} \mathrm{CrO}_{4}$ (Amersham, Germany), washed, and incubated for 60 minutes at $37^{\circ} \mathrm{C}$ with serial dilutions of patient sera, thereby using patient's complement. Release of ${ }^{51} \mathrm{Cr}$ from lysed target cells into the supernatant of the samples ("Cs") was measured using a $\gamma$-counter (Cobra 5005, Canberra-Packard, Australia). Spontaneous release ("Sr") and maximum release (100\%, "Mr") were measured after incubation of target cells with medium alone or with detergent ( $2 \%$ SDS), respectively. Cytotoxicity was calculated using the following formula $100 \% \times$ (Cs-Sr)/(Mr-Sr). The percentage of lysed cells was plotted against the logarithm of the IGN311 concentration 
$(\mathrm{ng} / \mathrm{ml})$.

\subsection{Antibody Dependent Cellular Cytotoxicity (ADCC)}

LeY positive SKBR3 tumor cells were incubated with $\mathrm{Na}_{2}{ }^{51} \mathrm{CrO}_{4}$ (Amersham, Germany), washed, and plated into 96-well microtiter plates. Effector cells derived from peripheral blood mononuclear cells from a healthy volunteer donor were freshly prepared and added to the target cells at an effector to target ratio of 40:1. Heat-inactivated serum samples were diluted 1:20 and incubated at $37^{\circ} \mathrm{C}$ for 16 hours in a $\mathrm{CO}_{2}$-incubator. Released ${ }^{51} \mathrm{Cr}$ indicative for target cell lysis was calculated using the procedure mentioned above.

\subsection{Determination of Anti Anti-Idiotypic Antibodies (Ab3)}

Antibody MMA383 was used to capture Ab3 antibodies in patient serum. Bound antibodies were detected with a human $\lambda$ light chain specific HRP conjugate. Sera were diluted $1: 10$.

\section{Results}

\subsection{HAHA Kinetic and Specificity}

The kinetic of the HAHA response in patient 1 (receiving $50 \mathrm{mg}$ IGN311) was determined by SPR using a quailfied Biacore method [15] (Figure 1(a)).

The observed, non-serious side effects during treatment were hyperuricaemia (grade 1) and epistaxis (grade 2). A significant HAHA response was detectable at day 8 and showed peak values at day 15 measured before the second infusion. Notably, the HAHA response decreased following the second infusion of IGN311 on day 15 and was also not re-boosted by a third and fourth application at days 108 and 123, respectively, indicative for the socalled HAHA type I [16]. The resolvement of the HAHA response over time suggests the establishment of tolerance. In comparison, patient 2 showed no response above background (data not shown). To determine the isotype of the bound immunoglobulin detected by SPR, patient 1 immune serum (day 15) was depleted from IgG by Protein-G affinity chromatography (Figure 1(b), left panel). Protein-G binds all human IgG subclasses with high affinity whereas $\operatorname{IgM}$ is not retained. The flow through after Protein-G affinity chromatography was collected and applied to BIACORE analysis. After Protein-G treatment no sample binding was observed (Figure 1(b), right panel) indicating that the HAHA response consisted of IgG only. To demonstrate whether the induced HAHA response is directed against the IGN311 binding site, an ELISA using mAb ABL364 - the murine IgG3/ $\kappa$

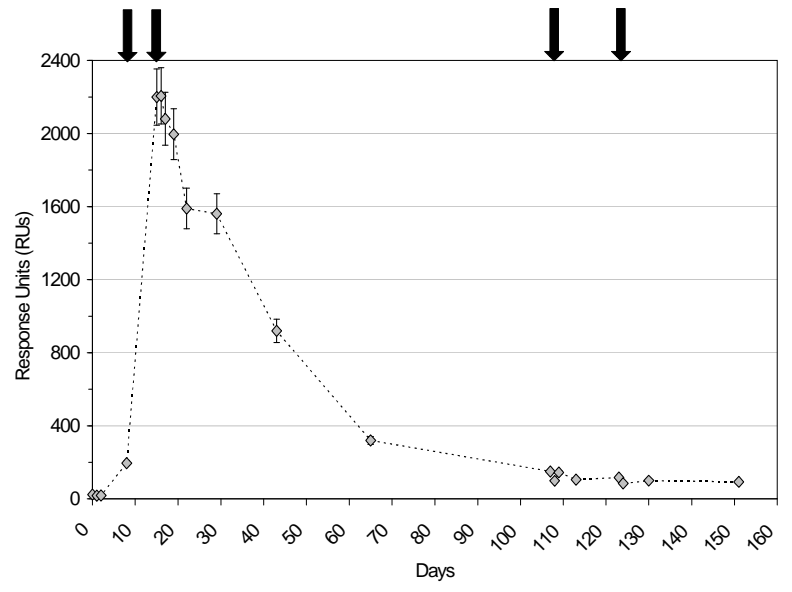

(a)
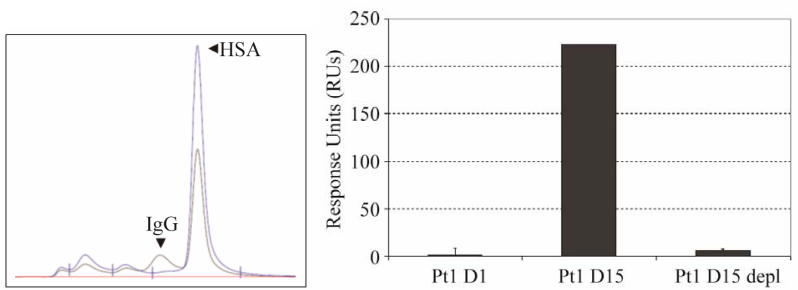

(b)

Figure 1. Kinetics of the HAHA reactivity induced in patient 1 (BIAcore). (a) Dose of $50 \mathrm{mg}$ IGN311 was applied to the patients at days $1,15,108$, and 123 (arrows). Blood was taken at days $0,1,8,15,17,19,22,29,43,65,108,109,113$, $123,130,151$, and 30 minutes after each application. Serum was prepared, diluted 1:10, and tested for binding to immobilized IGN311. The signal is recorded as relative Response Units (RU's); (b) Left panel: IgG depletion by Protein-G chromatography (SEC-HPLC). Histograms obtained with samples prior (black line) and after (blue line) column are shown as overlay. Based on the human serum albumin (HSA) peak (retention time $9.407 \mathrm{~min}$ ), the concentration was adjusted for the subsequent Biacore assay. Right panel: Determination of HAHA response (SPR). Binding of patient 1, day 1 (Pt1 D1) and day 15 (Pt1 D15) serum was compared to binding of depleted PT1 D15 serum.

variant of IGN311 - as coating antigen was performed. As control for reactivity against the murine Fc-region, an unrelated murine IgG3/ $/ \mathrm{mAb}$ was used. Pre-serum (day 1) and immune serum (day 15) of patient 1 were serially diluted and bound Abs were detected with an anti-human IgG-HRP conjugate.

A concentration-dependent binding of immune serum to ABL364, but not to the $\mathrm{IgG} 3 / \kappa$ isotype control antibody, was obtained (Figure 2(a)) indicating that the induced HAHA reactivity is idiotype-specific regarding IGN311. Isotype determination of the serum antibodies bound to ABL364 was performed with human IgG subclass-specific antibodies conjugated to HRP and total IgG was determined with polyclonal anti-human IgGHRP. Data shown in Figure 2(b) indicate that the in- 


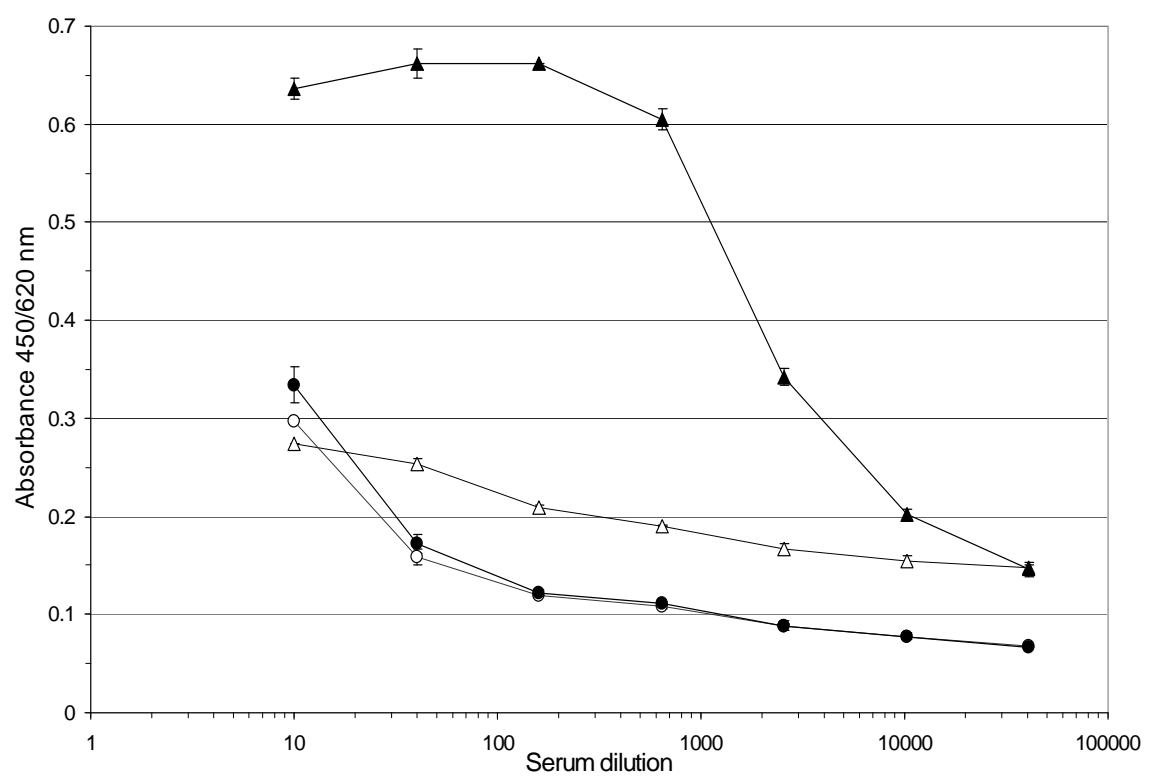

(a)

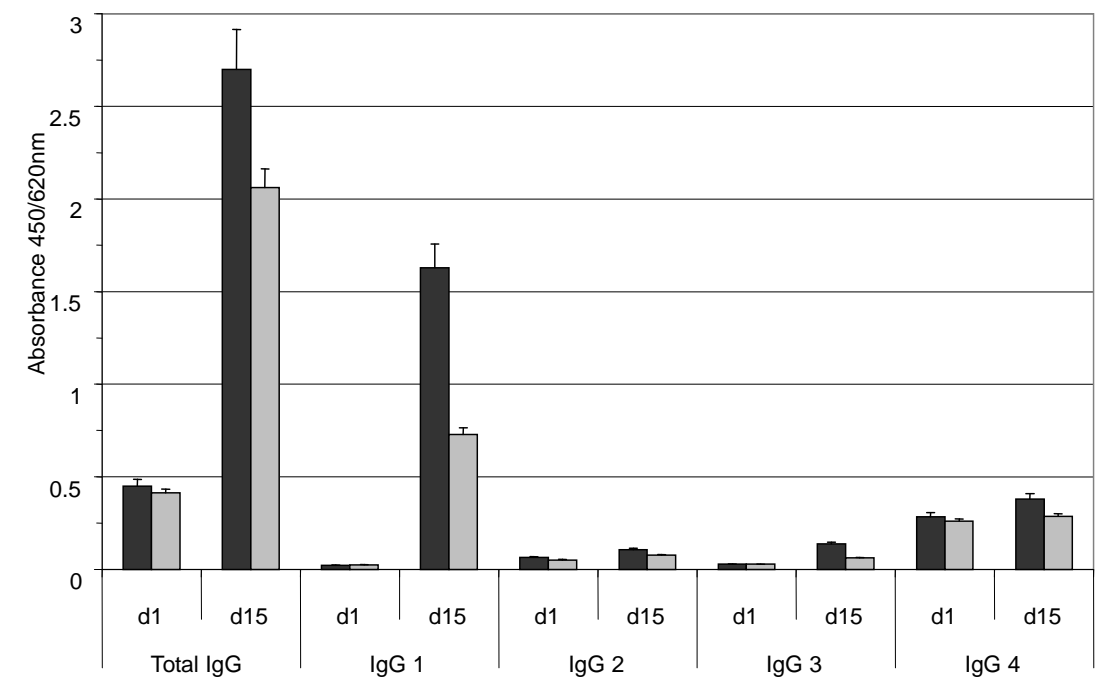

(b)

Figure 2. (a) Anti-Id ELISA. Binding of pre- (day 1, open triangles) and immune serum (day 15, black triangles) from patient 1 to ABL364 - the murine IgG3/ $\kappa$ variant of IGN311 - was measured. As isotype control, an unrelated murine IgG3/ (FLOPC-21, Sigma) was used (open circles: pre-serum, black circles: immune serum). Error bars indicate the arithmetic mean value of duplicate measurements; (b) Isotype determination of the anti-Id response (ELISA). Isotype determination of human serum antibodies bound to ABL364 was performed with human IgG subclass-specific antibodies conjugated to HRP (Sigma). As control, total IgG was determined with polyclonal anti-human IgG-HRP. Serum samples were diluted 1:300 (black bars) and 1:2700 (grey bars). Error bars indicate the arithmetic mean value of duplicate measurements.

duced HAHA response consisted largely of IgG1 antibodies. This is in accordance with results published by Ritter et al. [16] who showed that the isotype of the induced HAHA response after application of the humanized anti-EpCAM antibody A33 consisted of IgG1.

\subsection{Effector Mechanism}

Regarding the clinical effects, this patient showed nota- bly prolonged disease stabilization during the whole course of treatment. The underlying mechanism of antitumor activity leading to stabilization of disease in patient 1 was investigated by accessing the capability of the immune serum to lyse LeY expressing target cells by CDC (Figure 3(a)) and ADCC (Figure 3(b)). Interestingly, in patient 1 significant CDC activity could be detected only 30 minutes after the first infusion - whereas 


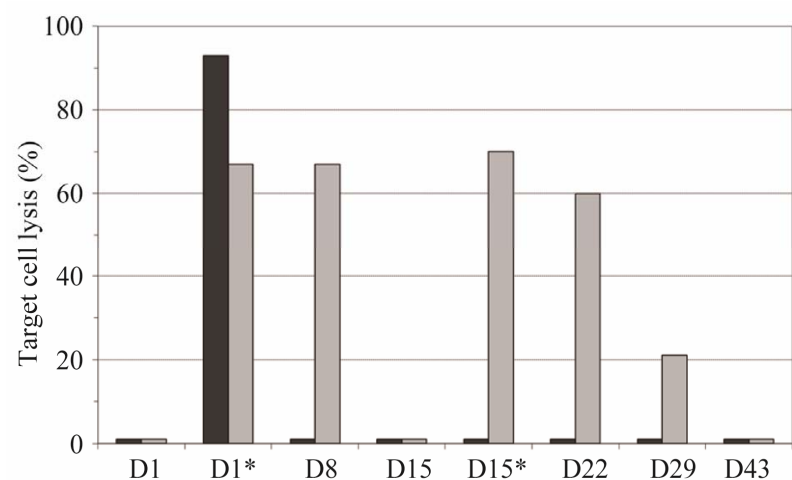

(a)

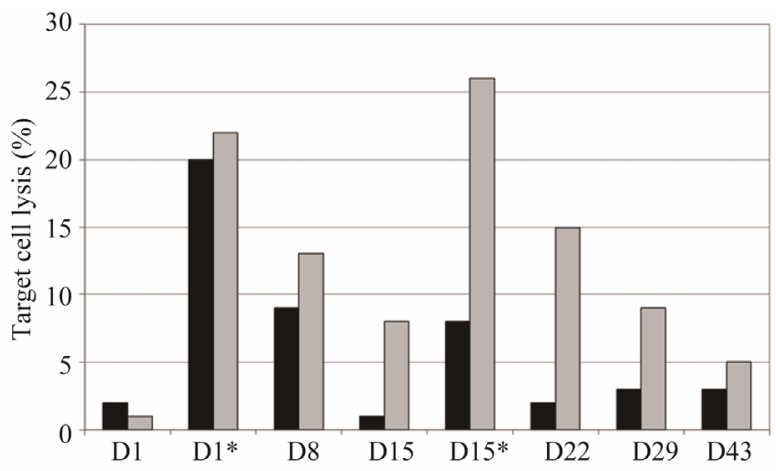

(b)

Figure 3. Comparison of cellular cytotoxicity (shown as percentage of lysed target cells) observed in the $50 \mathrm{mg}$ IGN311 dose group: CDC (a) and ADCC (b). Serum samples from days $1,8,15,22,29$, and 43 were tested-additionally samples taken 30 minutes after application of IGN311 at days 1 and 15 were measured (indicated by asterisk). Black bars: patient 1 , grey bars: patient 2 . ${ }^{*}$ Indicates time point after 2nd IGN311 infusion.

no reactivity was seen at any later time points tested. Most importantly, no CDC activity at all was detectable in patient 1 following the second infusion of IGN311. In contrast, patient 2 from the same dosage group, but without HAHA response, showed CDC reactivity during at least one week following both, the first as well as the second IGN311 infusion. This result strongly indicates that the induced HAHA response neutralizes the applied IGN311. Regarding the ADCC activity (Figure 3(b)), the proposed inhibitory effect of the HAHA response was less pronounced than observed for CDC activity. Nevertheless, when compared to patient 2, a reduction in ADCC was evident - particularly after the 2nd infusion at day 15 .

From these data it can be concluded that also ADCC is affected by the induced HAHA response although to a lower degree than the CDC. This may be explained by the fact that generally higher amounts of antibody are necessary for CDC compared to ADCC [18].

\subsection{Pharmacokinetics}

To investigate whether the amount of induced anti-IGN311 antibodies is, at least theoretically, sufficient for binding the LeY antigen, the IGN311 serum concentration during the treatment course was determined (Figure 4). Although IGN311 has shown a half-life in patient serum [14] of 10 - 12 days after the first infusion and of $\sim 24$ days following the second infusion of $100 \mathrm{mg}$ IGN-311/ patient [14], which is close to the reported $t 1 / 2$ of 21 days of natural $\mathrm{IgG}$, patient 1 showed a rather different picture.

Whereas after the 1st infusion, the pharmacokinetics (measured until day 8) were similar to the other two patients, a dramatic difference was evident 2 days after the 2nd infusion: in contrast to patient 2 whose IGN311 titer slowly decreased according to expected serum half-life, no IGN311 was detectable in patient 1 . Based on the presented data so far, the explanation for this observation is that IGN311 can form complexes with the idiotypespecific antibodies generated by the HAHA response and can therefore not be detected by ELISA based on an antiid antibody used for detection. In accordance with the rapid complex formation of IGN311 with the idiotype specific antibodies also the effector function are abrogated rapidly. To get information about how much anti-id antibodies were produced in patient 1 , we used the IGN311 anti-id antibody MMA383 as calibrator to quantitate the HAHA response (Table 1).

At peak levels (at day 15) around $15 \mu \mathrm{g} / \mathrm{ml}$ MMA383 equivalents are present in serum which are then steadily decreasing to approximately $6 \mu \mathrm{g} / \mathrm{ml}$ at day 65 . In contrast, a ten-fold lower amount was detected at day 8 indicating that this amount of HAHA response is most likely not sufficient to neutralize IGN311 (assuming a 1:1 stoichiometry). The measured amount of unbound IGN311 (Figure 4) correlated with the decrease in CDC and ADCC activity measured in the serum of patient 1 (Figures 3(a) and (b)).

\subsection{Ab3 Induction}

Regarding the correlation of the HAHA response with

Table 1. HAHA quantification. Using the murine mAb MMA383 as reference which recognizes the idiotype of IGN311 in a qualified BIACORE assay [14] the RU's were assigned to an amount of MMA383 equivalents $(\mu \mathrm{g} / \mathrm{ml})$.

\begin{tabular}{cc}
\hline Day & $\mu \mathrm{g} / \mathrm{ml}$ \\
\hline 1 & $\mathrm{bdl}$ \\
8 & 1.52 \\
15 & 15.16 \\
22 & 14.0 \\
65 & 5.78 \\
\hline
\end{tabular}




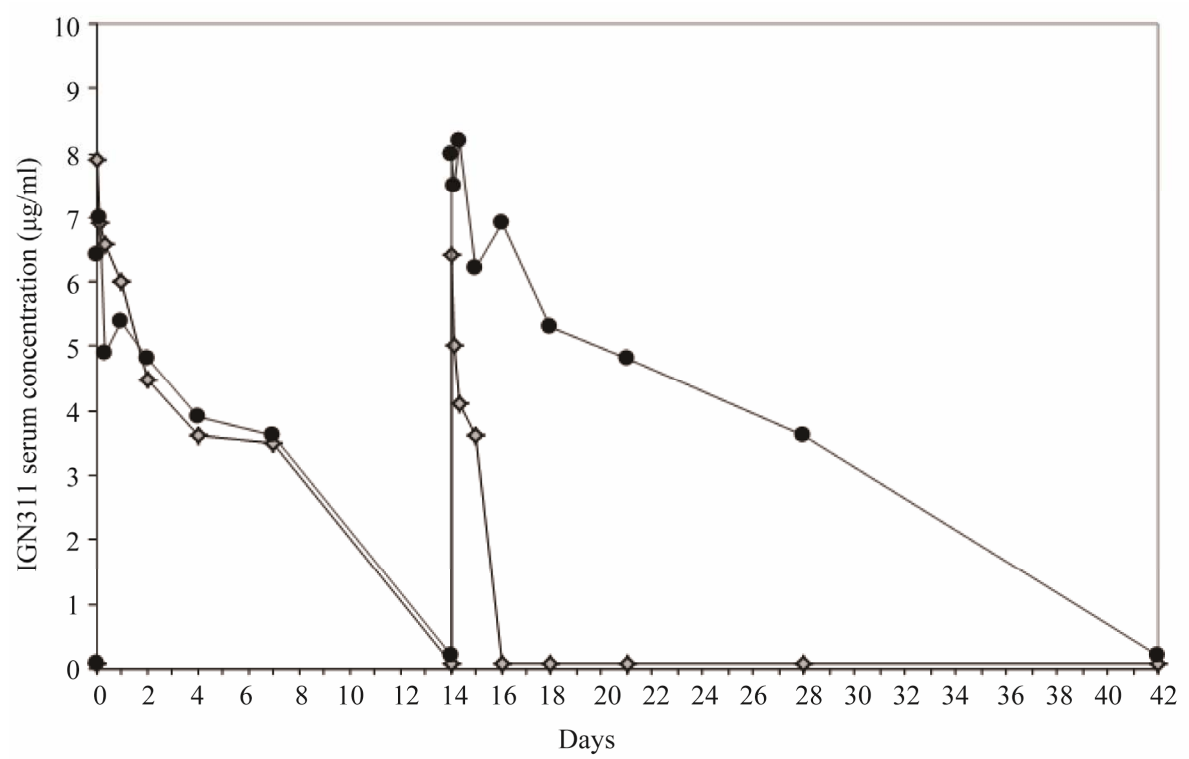

Figure 4. Determination of IGN311 levels in patient sera (ELISA). MMA383 was used to capture IGN311 present in patient serum at time points (hours) 0 (day 1, 1st infusion), 0.5, 4, 8, 24 (day 2), 48 (day 3), 96 (day 5), 168 (day 8), 336 (day 15, 2nd infusion), 336.5, 340, 344, 360 (day 16), 384 (day 17), 432 (day 19), 504 (day 22), 672 (day 29), and 1008 (day 43 ). Patient 1 (diamonds), patient 2 (circles).

the disease stabilization seen in patient 1 , we hypothesized that the induction of the anti-idiotypic HAHA (Ab2) response against IGN311 (Ab1) may consequently result in generation of anti-anti-idiotypic antibodies (Ab3) [19, 20] which recognize LeY on the target cells leading to an in situ immunization against the per se non-immu-nogenic carbohydrate LeY target antigen [18]. Therefore, we used an ELISA approach to verify induction of Ab3 antibodies. False positive signals resulting from remaining serum IGN311 $(\operatorname{IgG} 1 / \kappa)$ were eliminated by an ELISA detection system specific for the human $\lambda$ chain. In humans, the ratio of antibodies containing $\kappa$ or $\lambda$ light chains is $60: 40$ [21] therefore the chance for the existence of a specific Ab3 clone expressing a $\lambda$ light chain or $\lambda$ light chain is roughly equal. As can be seen in Figure 5, patient 1 developed a significant $\lambda$ chain related anti-id (antiMMA383) reactivity.

\section{Discussion}

The induction of an immune response against passively administered murine therapeutic antibodies (HAMA) has been frequently reported and was found to be associated with severe side effects and unfavorable pharmacokinetics. On the other hand, HAMA can also be associated with a positive clinical outcome [22] because-apart from direct cytotoxic mechanisms such as ADCC, CDC and induction of apoptosis-Ab2 and Ab3 have been postulated according to the network hypothesis of Jerne to induce tumour cell rejection $[3,23]$. According to Jerne's network theory, a subset of anti-idiotypic Abs (Ab2beta) carries an "internal image" of the $\mathrm{Ag}$ and induces $\mathrm{Abs}$ (Ab3) against the original Ag [Jerne, 1974]. Whereas an immune response against murine Abs can be directed against all parts of the molecule which are non-homologous to their human counterparts, humanized mAbs should only be immunogenic with regard to the murine amino acid sequences in their antigen binding site. Consequently, a human anti-human antibody (HAHA) response should be idiotype-specific. Interestingly, investigations regarding the induced HAHA responses in clinical trials revealed that application of different humanized antibodies (although all $\mathrm{IgG} 1 / \kappa$ ) induced different levels of HAHA [6]. Whereas passive application of Herceptin or Avastin induced marginal levels of HAHA [24,25], application of huA33 [26] resulted in greater than $50 \%$ HAHA responders indicating that a) humanization does not resolve all immunogenicity issues and that $b$ ) the occurrence of HAHA response cannot be predicted. The high incidence of HAHA induction by a humanized antibody huA33 was also shown by the data obtained with IGN311. Additionally, the high frequency of HAHA was in all three cases measured by a surface plasmon resonance approach and not by ELISA. This finding warranted a side-by-side comparison of both techniques to assess the possibility of identifying false positive/false negative HAHA responders. Such approach was taken by Lofgren et al. [27] and indeed they identified more HAHA positives samples when applying SPR technology.

Regarding an anti-anti-idiotypic response, Cheung et 


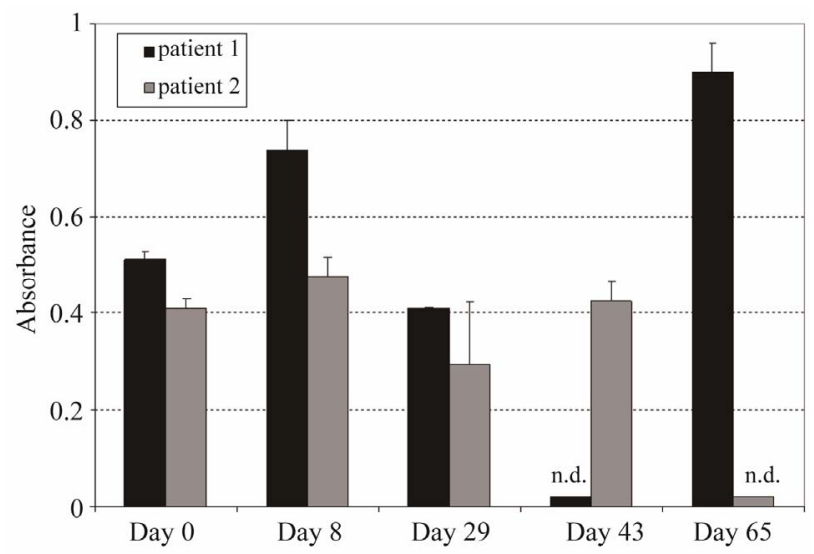

Figure 5. Determination of Ab3 antibodies after IGN311 (Ab1) application (ELISA). MMA383 was used to capture Ab3 antibodies in patient serum. Sera were diluted 1:10 and bound antibodies were detected with a human $\lambda$ light chain specific HRP conjugate. Values shown have been blanked against day 0 . For graphical depiction, day 0 was set to an absorbance value of 0.05 . $n . d=$ not determined.

al. [28] showed that Ab3 antibodies are induced after application of the anti-GD2 mAb 3F8. Furthermore, Ab3 induction was associated with long term survival supporting the network concept postulated by Jerne. The finding that high HAMA titers, resulting in IgG Ab3 antibodies, prolong survival, are consistent with the finding that IGN311 treated patient 1 showed stable disease over the whole treatment period and post-treatment observation period. Uttenreuther-Fischer et al. [23] analyzed the details of the immune response in a neuroblastoma patient also with molecular methods and found that the idiotypic $\mathrm{Ab}(\mathrm{Ab} 1)$ against the target GD2, a disialoganglioside overexpressed in neuroblastoma cells triggered the production of an anti-idiotypic $\mathrm{Ab}(\mathrm{Ab} 2)$. This $\mathrm{Ab} 2$ belonged to the Ab2beta subclass, which means that it mirrors the spatial structure of the Ag recognized by the therapeutic antibody (Ab1). Most importantly, this Ab2beta was capable of inducing the production of an anti-anti-idiotypic antibody, designated Ab3. This Ab3 in turn was able to bind the original tumor-associated antigen, resulting in a secondary immune response against the tumor cells. According to the authors, Ab3 production started with a delay of $6-14$ months relative to the first application of the initial therapeutic mAb but then the production persisted for years, significantly contributing to the long-lasting, positive effect of the $\mathrm{Ab}$ therapy. Additionally to its beneficial effect in the patient, Ab3 could also be used as a surrogate marker for the success of the immune-therapeutic intervention.

The present data show that patient 1 receiving IGN311 (lowest dose, $50 \mathrm{mg} / \mathrm{kg}$ ) developed a $\lambda$ chain related anti-idiotypic (anti-MMA383) immune response. The ob- served long-term benefit from the immune therapy was presumably also, at least partly, mediated by the mechanisms originally described by Uttenreuther-Fischer et al. This is also supported by the finding that the blood-levels of Ab1 in patient 1 decreased very quickly after the second $\mathrm{Ab}$ injection, a hint that production of the antiidiotypic antibody resulted in a fast clearance of the therapeutic antibody. The rapid clearance of the antibody might also explain the reduced ability of the serum of patient 1 to induce immunogenic effects in the CDC and ADCC assays. Importantly, Ab3 is supposed to directly recognize LeY modified epitopes on the targeted tumor cells, thereby triggering additional effector functions in situ and even possibly facilitates the induction of an active immune response that specifically attacks these malignant cells for long term. This proposed effect presumably also contributes to the improved long-term outcome of IGN311 therapy seen in patient 1 , despite a clearly compromised CDC and ADCC reactivity measured in the serum from this patient shortly after application of the therapeutic antibody. The induction of the anti-idiotypic responses following passive application of therapeutic antibodies may induce an immunization against the original antigen, by mimicking the original antigen by a part of the anti-idiotypic antibodies. Such active immunization component may prolong significantly the therapeutic effects triggered by the passive application of therapeutic antibodies. Moreover, in particular also for low immunogenic antigens such as carbohydrate antigens (including LeY) which are not able to induce an efficient immune response by themselves the mimicking image presentation by the anti-idiotypic antibodies can significantly enhance the induction of effective immune responses against the original antigen. Furthermore, it was also shown that the binding of IGN311 to LeY-modified receptors induced an inhibition of signal transduction via these receptors [29]. This mechanism additionally enhanced the effect of the immunotherapy because co-expression of LeY antigen and of EpCAM receptors results in a poor prognosis for these patients [30].

\section{Conclusion}

The presented data shed some light on the immunological basis for the long-term clinical disease stabilization found in one patient who developed a pronounced HAHA type I response after infusion of the humanized antibody IGN311. This also encourages further research to clarify the exact molecular mechanisms underlying the described effects. In the light of the recently published literature, this might help to further improve the study design and implementation of future clinical trials, resulting in a less burdening treatment and a positive long- 
term outcome in treated patients by inducing an active immunization component following passive application of therapeutic antibodies [31].

\section{Acknowledgements}

A. N. and R. K. thank Dr. Gerald Schmid for manuscript review.

\section{REFERENCES}

[1] A. Nechansky and R. Kircheis, "Immunogenicity of Therapeutics: A Matter of Efficacy and Safety," Expert Opinion Drug Discovery, Vol. 5, No. 11, 2010, pp. 1-13.

[2] M. B. Khazaeli, R. M. Conry and A. F. LoBuglio, "Human Immune Response to Monoclonal Antibodies," Journal Immunotherapy Emphasis Tumor Immunology, 1994, Vol. 15, No. 1, pp. 42-52. doi:10.1097/00002371-199401000-00006

[3] J. E. Frodin, M. E. Faxas, B. Hagstrom, A. K. Lefvert, G. Masucci, B. Nilsson, M. Steinitz, P. Unger and H. Mellstedt, "Induction of Anti-Idiotypic (ab2) and Anti-AntiIdiotypic (ab3) Antibodies in Patients Treated with the Mouse Monoclonal Antibody 17-1A (ab1). Relation to the Clinical Outcome-An Important Antitumoral Effector Function?" Hybridoma, Vol. 10, No. 4, 1991, pp. 421431. doi:10.1089/hyb.1991.10.421

[4] H. Koprowski, D. Herlyn, M. Lubeck, E. DeFreitas and H. F. Sears, "Human Anti-Idiotype Antibodies in Cancer Patients: Is the Modulation of the Immune Response Beneficial for the Patient?" Proceedings of the National Academy of Sciences of the USA, Vol. 81, No. 1, 1994, pp. 216-219. doi:10.1073/pnas.81.1.216

[5] O. H. Brekke and I. Sandlie, "Therapeutic Antibodies for Human Diseases at the Dawn of the Twenty-First Century," Nature Reviews Drug Discovery, Vol. 2, No. 3, 2003, pp. 52-62. doi:10.1038/nrd984

[6] A. Nechansky, "HAHA - Nothing to Laugh about. Measuring the Immunogenicity (Human Anti-Human Antibody Response) Induced by Humanized Monoclonal Antibodies Applying ELISA and SPR Technology," Journal of Pharmaceutical and Biomedical Analysis, Vol. 51, No. 1, 2010, pp. 252-254. doi:10.1016/j.jpba.2009.07.013

[7] J. Fagerberg, A. L. Hjelm, P. Ragnhammar, J. E. Frodin, H. Wigzell and H. Mellstedt, "Tumor Regression in Monoclonal Antibody-Treated Patients Correlates with the Presence of Anti-Idiotype-Reactive T Lymphocytes," Cancer Research, Vol. 55, No. 9, 1995, pp. 1824-1827.

[8] R. Gruber, L. J. van Haarlem, S. O. Warnaar, E. Holz and G. Riethmuller, "The Human Antimouse Immunoglobulin Response and the Anti-Idiotypic Network Have No Influence on Clinical Outcome in Patients with Minimal Residual Colorectal Cancer Treated with Monoclonal Antibody CO17-1A," Cancer Research, Vol. 60, No. 7, 2000, pp. 1921-1926.

[9] J. Ma, J. Samuel, G. S. Kwon, A. A. Noujaim and R. Madiyalakan, "Induction of Anti-Idiotypic Humoral and Cellular Immune Responses by a Murine Monoclonal
Antibody Recognizing the Ovarian Carcinoma Antigen CA125 Encapsulated in Biodegradable Microspheres," Cancer Immunology Immunotherapy, Vol. 47, No. 1, 1998, pp. 13-20. doi: $10.1007 / \mathrm{s} 002620050499$

[10] D. Herlyn, R. Somasundaram, W. Li and H. Maruyama, "Anti-Idiotype Cancer Vaccines: Past and Future," Cancer Immunology Immunotherapy, Vol. 43, No. 2, 1996, pp. 65-76. doi: $10.1007 / \mathrm{s} 002620050305$

[11] Y. Shoenfeld, "The Idiotypic Network in Autoimmunity: Antibodies That Bind," Nature Medicine, Vol. 10, No. 1, 1994, pp. 17-18.

[12] Y. Shoenfeld, H. Amital, S. Ferrone and R.C. Kennedy, "Anti-Idiotypes and Their Application under Autoimmune, Neoplastic, and Infectious Conditions," International Archives Allergy Immunology, Vol. 105, No. 3, 2005, pp. 211-223. doi:10.1159/000236760

[13] N. K. Jerne, "Towards a Network Theory of the Immune System," Annual Immunology, Vol. 125, No. 1-2, 1974, pp. 373-389.

[14] D. V. Oruzio, G. Waxenecker, C. Aulmann, B. Märkl, T. Wagner, G. C. Mudde, M. Schuster, N. Eller, A. Mayer, S. Stranner, G. Himmler, H. Loibner, G. Schlimok, R. Kircheis and A. Nechansky, "Phase I Dose Escalation Study with Le Y Carbohydrate Specific Humanized mAb IGN311," Journal Cancer Therapy, Vol. 2, 2011, pp. 760-771. doi:10.4236/jct.2011.25102

[15] O. Szolar, S. Stranner, I. Zinoecker, G. C. Mudde, G. Himmler, G. Waxenecker and A. Nechansky, "Qualification and Application of a Surface Plasmon ResonanceBased Assay for Monitoring Potential HAHA Responses Induced after Passive Administration of a Humanized Anti Lewis-Y Antibody," Journal of Pharmaceutical and Biomedical Analysis, Vol. 41, No. 4, 2006, pp. 13471353. doi:10.1016/j.jpba.2006.03.026

[16] G. Ritter, L. S. Cohen, C. Williams, E. C. Richards Jr., L. J. Old and S. Welt , "Serological Analysis of Human Anti-Human Antibody Responses in Colon Cancer Patients Treated with Repeated Doses of Humanized Monoclonal Antibody A33," Cancer Research, Vol. 61, No. 18, 2001, pp. 6851-6859.

[17] M. S. Co, J. Baker, K. Bednarik, E. Janzek, W. Neruda, P. Mayer, R. Plot, B. Stumper, M. Vasquez, C. Queen and H. Loibner, "Humanized Anti-Lewis Y antibodies: In Vitro Properties and Pharmacokinetics in Rhesus Monkeys," Cancer Research, Vol. 56, No. 5, 1996, pp. 1118-1125.

[18] M. Schuster, P. Umana, C. Ferrara, P. Brunker, C. Gerdes, G. Waxenecker, S. Wiederkum, C. Schwager, H. Loibner, G. Himmler and G. C. Mudde, "Improved Effector Functions of a Therapeutic Monoclonal Lewis Y-specific Antibody by Glycoform Engineering," Cancer Research, Vol. 65, No. 17, 2005, pp. 7934-7941.

[19] N. K. Cheung, I. Y. Cheung, A. Canete, S. J. Yeh, B. Kushner, M. A. Bonilla, G. Heller and S. M. Larson, "Antibody Response to Murine Anti-GD2 Monoclonal Antibodies: Correlation with Patient Survival," Cancer Research, Vol. 54, 1994, pp. 2228-2233.

[20] J. Fagerberg, P. Ragnhammar, M. Liljefors, A. L. Hjelm, H. Mellstedt and J. E. Frodin, "Humoral Anti-Idiotypic 
and Anti-Anti-Idiotypic Immune Response in Cancer Patients Treated with Monoclonal Antibody 17-1A," Cancer Immunology Immunotherapy, Vol. 42, No. 2, 1996, pp. 81-87. doi:10.1007/s002620050255

[21] A. V. Popov, X. Zou, J. Xian, I. C. Nicholson and M. Bruggemann, "A Human Immunoglobulin Lambda Locus Is Similarly Well Expressed in Mice and Humans," Journal Experimental Medicine, Vol. 189, No. 10, 1999, pp. 1611-1620. doi:10.1084/jem.189.10.1611

[22] J. Fagerberg, J. E. Frodin, P. Ragnhammar, M. Steinitz, H. Wigzell and H. Mellstedt, "Induction of an Immune Network Cascade in Cancer Patients Treated with Monoclonal Antibodies (ab1). Is Induction of Anti-Idiotype Reactive T Cells (T3) of Importance for Tumor Response to mAb Therapy?" Cancer Immunol Immunotherapy, Vol. 38, 1994, pp. 149-159. doi:10.1007/s002620050048

[23] M. M. Uttenreuther-Fischer, J. A. Kruger and P. Fischer, "Molecular Characterization of the Anti-Idiotypic Immune Response of a Relapse-Free Neuroblastoma Patient Following Antibody Therapy: A Possible Vaccine against Tumors of Neuroectodermal Origin?" Journal Immunology, Vol. 176, No. 12, 2006, pp. 7775-7786.

[24] M. A. Cobleigh, C. L. Vogel, D. Tripathy, N. J. Robert, S. Scholl, L. Fehrenbacher, J. M. Wolter, V. Paton, S. Shak, G. Lieberman and D. J. Slamon, "Multinational Study of the Efficacy and Safety of Humanized Anti-HER2 Monoclonal Antibody in Women Who Have HER2-Overexpressing Metastatic Breast Cancer That Has Progressed after Chemotherapy for Metastatic Disease," Journal Clinical Oncology, Vol. 17, No. 9, 1999, pp. 2639-2648.

[25] G. Hale, P. Rebello, L. R. Brettman, C. Fegan, B. Kennedy, E. Kimby, M. Leach, J. Lundin, H. Mellstedt, P. Moreton, A. C. Rawstron, H. Waldmann, A. Osterborg and P. Hillmen, "Blood Concentrations of Alemtuzumab and Antiglobulin Responses in Patients with Chronic Lymphocytic Leukemia Following Intravenous or Subcutaneous Routes of Administration," Blood, Vol. 104, 2004, pp. 948-955. doi:10.1182/blood-2004-02-0593

[26] S. Welt, G. Ritter, C. Williams Jr, L. S. Cohen, M. John, A. Jungbluth, E. A. Richards, L. J. Old and N. E. Kemeny, "Phase I Study of Anticolon Cancer Humanized Antibody A33," Clinical Cancer Research, Vol. 9, No. 4, 2003, pp. 1338-1346.

[27] J. A. Lofgren, S. Dhandapani, J. J. Pennucci, C. M. Abbott, D. T. Mytych, A. Kaliyaperumal, S. J. Swanson and M. C. Mullenix, "Comparing ELISA and Surface Plasmon Resonance for Assessing Clinical Immunogenicity of Panitumumab," Journal Immunology, Vol. 178, No. 11, 2007, pp. 7467-7472.

[28] N.-K. V. Cheung, H. F. Guo, G. Heller and I. Y. Cheung, "Induction of Ab3 and Ab3' Antibody Was Associated with Long-Term Survival after Anti-G(D2) Antibody Therapy of Stage 4 Neuroblastoma," Clinical Cancer Research, Vol. 6, 2000, pp. 2653-2660.

[29] M. Klinger, H. Farhan, H. Just, H. Drobny, G. Himmler, G. C. Mudde, M. Freissmuth and V. Sexl, "Antibodies Directed against Lewis-Y Antigen Inhibit Signaling of Lewis-Y Modified ErbB Receptors," Cancer Research, Vol. 64, No. 3, 2007, pp. 1087-1093. doi:10.1158/0008-5472.CAN-03-2435

[30] G. Sauter, R. Simon and K. Hillan, "Tissue Microarrays in Drug Discovery," Nature Reviews Drug Discovery, Vol. 2, No. 12, 2003, pp. 962-972. doi:10.1038/nrd1254

[31] M. Schuster, A. Nechansky, H. Loibner and R. Kircheis, "Cancer Immunotherapy," Biotechnol Journal, Vol. 1, No 2, 2006, pp. 138-147. doi:10.1002/biot.200500044 